Special issue of the 2nd International Conference on Computational and Experimental Science and Engineering (ICCESEN 2015)

\title{
CRI and GAI Comparison in LED Light Sources Using Image Processing
}

\author{
İ.S. ÜNCÜ ${ }^{a, *}$, S. CoşKUnsu ${ }^{b}$, C. SÖNMEZ ${ }^{a}$ AND O. BIÇAKÇI ${ }^{c}$ \\ ${ }^{a}$ Süleyman Demirel University, Technology Faculty, Electrical and Electronic Engineering Department, \\ Isparta, Turkey \\ ${ }^{b}$ Süleyman Demirel University, Engineering Faculty, Computer Engineering Department, \\ Isparta, Turkey \\ ${ }^{c}$ AğustosTeknoloji LTD., İstanbul, Turkey
}

\begin{abstract}
In this study, color rendering index and gamut area index related to the light quality of a light source are compared. Color rendering index is a measure that demonstrates how much a light source reveals colors in nature. In color rendering index measurements, average of reference colors is taken to obtain an overall measurement value. As to gamut area index measurement, the result is obtained through the field measurement formed by the results of reference colors in the color space.
\end{abstract}

DOI: 10.12693/APhysPolA.130.379

PACS/topics: 07.05.Pj, 42.30.-d, 42.66.Ne

\section{Introduction}

The main objective in the measurement of color rendering of a lighting product is to calculate to what extent it can reveal the accurate colors of an object. In 1947, P.S. Bouma has explained the characteristics of an artificial light source from the perspective of the daylight: (1) it should show the great diversity of colors, (2) it should enable to distinguish color tones from each other and (3) it should ensure that the surrounding objects look completely natural [1]. In 1965, color rendering index (CRI) which determines the impact of the light source on colors was developed by Nickerson and Jerome. It was standardized by CIE (Commission Internationale

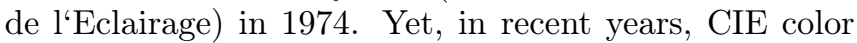
rendering metrics of the psychophysical experiments have been observed to have a poor correlation in a variety of LED light sources [2]. In the early 1970s, Thornton has developed the concept of gamut area index (GAI) alternative to color rendering measurement method. In his study, Thornton has tried to detect differences in tones and vitality of the colors. Recently, both CRI and GAI are used to determine color rendering. The rationale of this approach is that CRI and GAI are totally different from each other, but both are related to the color rendering. While CRI value emphasizes the color stability compared to the known light source, GAI value lays emphasis on tones and vitality of colors $[3,5]$.

The use of cameras has been frequently applied in various measurement and error control systems. One of these systems is photometric measurements. It has also become possible to do multiple measurements on the same photo with the use of cameras used in photometric

\footnotetext{
*corresponding author; e-mail: serkanuncu@sdu.edu.tr
}

measurements [6]. Likewise in this study, CRI and GAI measurement was conducted on the same photo with different software.

LED lighting luminaires with different colour temperatures and different CRI values were investigated in this study. LED products used in this study and their features are shown in Table I.

Photographing pool was formed for measurements not to be affected by the ambient light. With this pool, it is aimed to reduce the error margin of measurement by creating a standard environment for LEDs with different color temperatures. In addition, a step motor controlled mechanism was constructed to ensure view of the objects to be photographed from every angle in the lighting pool. Lighting pool is shown in Fig. 1.

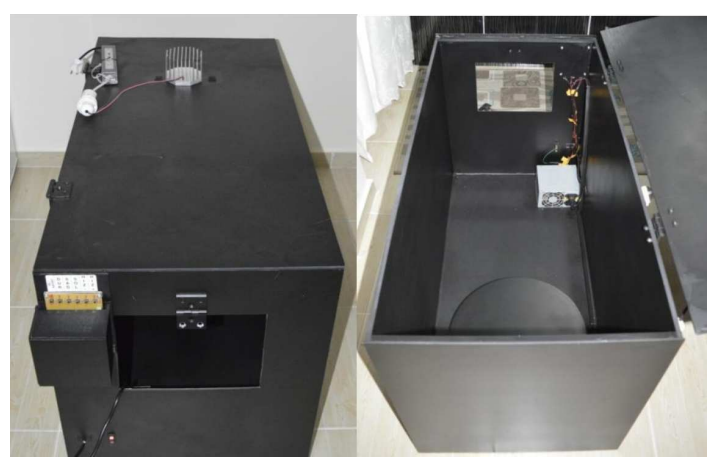

Fig. 1. Lighting pool.

To view the effect of LED lighting elements with different color temperatures on colors in the lighting pool, the same flower is photographed under the light of different color temperatures. Change on the colors induced by the studied lighting products, described by their code letters, is shown in Fig. 2. 
TABLE I

LED lighting products.

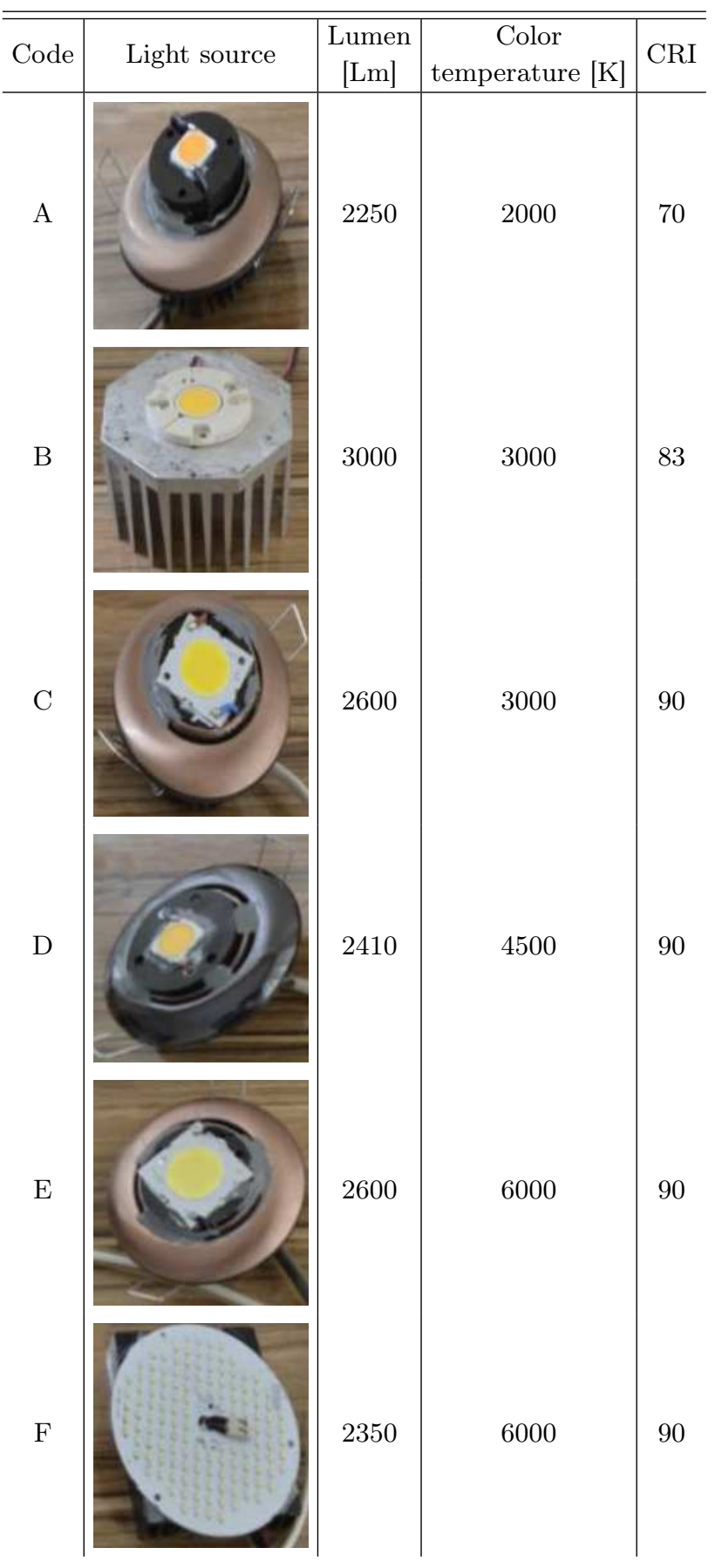

Two different software interfaces were developed using $\mathrm{C} \#$ programming language in order to measure CRI and GAI values. In both of the software, CRI and GAI values are calculated with image processing methods by using the specified colours ranging R1-R8 in CIE 13.3-1995 method. LEDs with $3000 \mathrm{~K}$ color temperature are used in both of the software interfaces given in Fig. 3. As can be observed in both interfaces, CRI and GAI values are very close to each other.

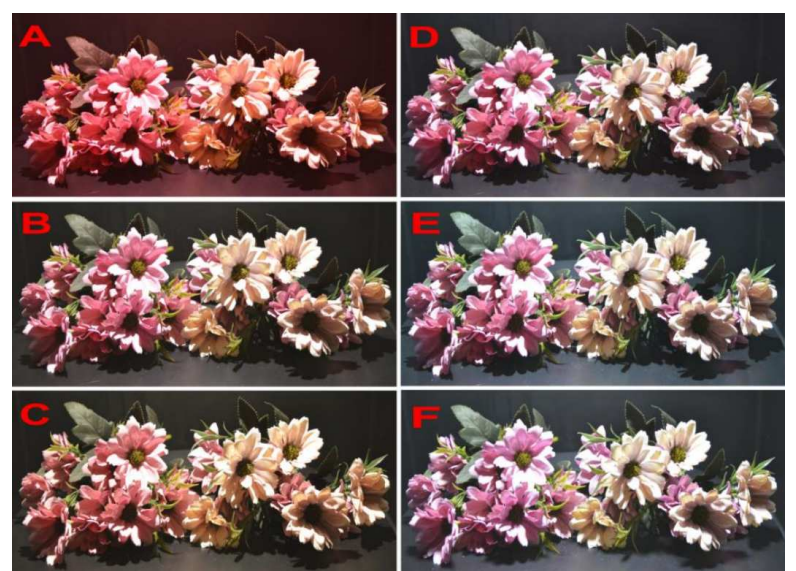

Fig. 2. Flower image illuminated by LEDs with different color temperatures.

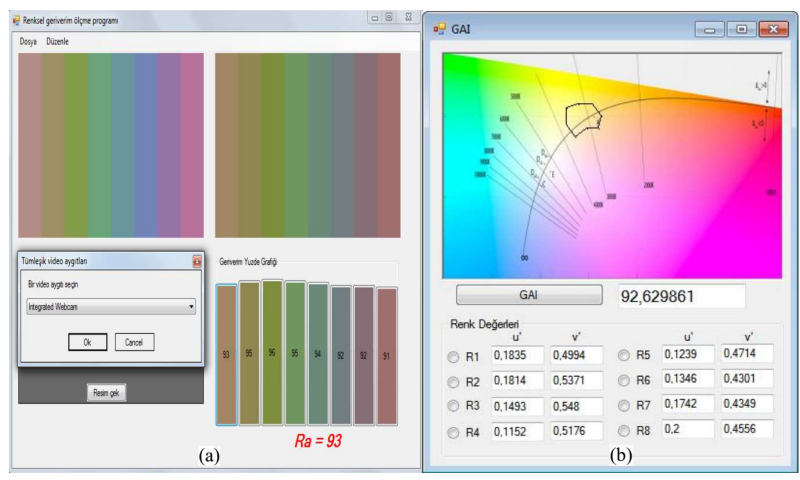

Fig. 3. Developed software interfaces, (a) CRI calculation software, (b) GAI calculation software.

\section{Conclusions}

CRI value of a lamp with $3000 \mathrm{~K}$ color temperature is calculated to be 93, and GAI value is measured as 92.62 in the prepared software. Yet, CRI and GAI measurements of the same lighting luminaires can be different. In their study, Rea et al. have observed that CRI and GAI measurements of the same lighting luminaires differ greatly [1]. Table II shows the values of that study.

Therefore, as emphasized in their study, Rea [1], ASSIST [4] and Freyssinier [3] indicate that natural and vivid lighting can be achieved if both $\mathrm{CRI}>80$ and $80 \leq$ GAI $\leq 100$ are provided, when selecting the light sources. Furthermore, along with this study, the system that finds GAI and CRI measurements of the photo through image processing algorithms has been developed. Therefore, a new area for image processing and the use of camera as measurement equipment has emerged.

\section{References}

[1] M.S. Rea, J.P. Freyssinier-Nova, Color Res. Appl. 33, 192 (2008).

[2] K. Smet, W.R. Ryckaert, M.R. Pointer, G. Deconinck, P. Hanselaer, Opt. Express 19, 8151 (2011). 
TABLE II

Color characteristics of the white illuminants used in Rea's study [1].

\begin{tabular}{c|c|c|c}
\hline \hline LightsSource & CCT [K] & CRI & GAI \\
\hline WW1-5 fc & 3157 & 95 & 52 \\
WW1-50 fc & 3174 & 95 & 55 \\
WW2-5 fc & 3399 & 80 & 64 \\
WW2-50 fc & 3443 & 80 & 64 \\
WW3b-5 fc ${ }^{a}$ & 3707 & 81 & 73 \\
WW3b-50 fc $^{a}$ & 3732 & 81 & 74 \\
WW4-5 fc & 3279 & 92 & 94 \\
WW4-50 fc & 3261 & 91 & 93 \\
CW1-5 fc & 5069 & 75 & 65 \\
CW1-50 fc & 5137 & 75 & 66 \\
CW2-5 fc & 6502 & 78 & 95 \\
CW2-50 fc & 6682 & 78 & 97 \\
CW3-5 fc & 6400 & 72 & 81 \\
CW3-50 fc & 6126 & 71 & 81 \\
CW4-5 fc & 5239 & 94 & 90 \\
CW4-50 fc & 5854 & 94 & 99
\end{tabular}

[3] J.P. Freyssinier, M. Rea, in: Proc. SPIE 7784, Tenth International Conference on Solid State Lighting, International Society for Optics and Photonics, San Diego 2010.

[4] Alliance for Solid-State Illumination Systems and Technologies ASSIST, Recommendations for Specifying Color Properties of Light Sources for Retail Merchandising, Vol. 8, Lighting Research Center, Troy (NY) 2010.

[5] İ.S. Üncü, İ. Taşc1, in: 8th National Lighting Congress, İstanbul 2011, p. 63.

[6] İ.S. Üncü, S. Coşkunsu, Acta Phys. Pol. A 128, B471 (2015). 